\title{
Glycerol Electrooxidation on Platinum-Tin Electrodeposited Films: Inducing Changes in Surface Composition by Cyclic Voltammetry
}

\author{
Gisele A. B. Mello ${ }^{1}$ - Pablo S. Fernández ${ }^{2}$ - María E. Martins ${ }^{3}$ - Giuseppe A. Camara ${ }^{1}$
}

(C) Springer Science+Business Media New York 2016

\begin{abstract}
In this work, PtSn binary electrodeposits were prepared in three compositions and submitted to successive voltammetric cycles in presence of glycerol $\left(1.0 \mathrm{~mol} \mathrm{~L}^{-1}\right)$ in acidic media. Catalysts were characterized by energy dispersive X-ray analysis and X-ray photoelectron spectroscopy before and after the cycles being performed, in order to check eventual changes in their compositions during the process. Spectroscopic results show that surface compositions are sensibly richer in Sn than their bulk counterparts. Overall, PtSn catalysts show a poor initial catalytic activity toward glycerol electrooxidation. However, as the cycles succeed, the voltammetric responses increasingly resemble that of $\mathrm{Pt}$, while the oxidation currents increase. Results are rationalized in terms of a continuous enrichment of the surface by $\mathrm{Pt}$ at the expenses of a loss of Sn. Moreover, when the electrochemical surface area (ECSA) is estimated by stripping of $\mathrm{CO}$, it becomes evident that electrooxidation currents remain growing, even when the ECSA is decreased, which makes the gain in catalytic activity particularly relevant. Ultimately, from a broader perspective, our results suggest that catalytic surfaces with tunable features (such as surface composition and catalytic response) can be obtained by the application of easily executable electrochemical protocols.
\end{abstract}

Giuseppe A. Camara

giuseppe.silva@ufms.br

1 Institute of Chemistry, Universidade Federal de Mato Grosso do Sul, 549, Campo Grande, MS 79070-900, Brazil

2 Instituto de Química, Universidade Estadual de Campinas, Cidade Universitária “Zeferino Vaz", Barão Geraldo,

Campinas, SP 13083-970, Brazil

3 Facultad de Ciencias Exactas, UNLP, CCT La Plata-CONICET, Instituto de Investigaciones Fisicoquímicas Teóricas y Aplicadas (INIFTA), 1900 La Plata, Argentina
Keywords Glycerol electrooxidation · Platinum-tin · Electrodeposits · Tunable composition · Surface composition change

\section{Introduction}

Glycerol is a polyol that has several interesting properties that make it a candidate for feeding direct alcohol fuel cells. Among these, it is worth noting the decreasing international prices (due to recent market surpluses) [1], non-flammability [1], nontoxicity [1], and relatively high-energy content (theoretically, 14 electrons are available per molecule) [2]. However, in order to convert glycerol in a potential fuel for anodes in DAFCs, an important technological drawback must still be surpassed. Because of its relative molecular complexity, glycerol is hard to be oxidized to $\mathrm{CO}_{2}$ on platinum surfaces (the quintessential catalyst for DAFCs) and during the electrocatalytic process most molecules are partially oxidized, generating a wide range of side products and reaction intermediates [2-4]. In a seminal paper, Lamy et al., by using HPLC, detected glyceraldehyde, glyceric acid, and other substances on Pt in both acid and alkaline media [4]. More recently, our group demonstrated that important amounts of $\mathrm{CO}_{2}$ are produced when glycerol is oxidized on $\mathrm{Pt}$, providing that the potential is higher than $0.9 \mathrm{~V}$ vs. reversible hydrogen electrode (RHE) [5, 6]. Unfortunately, the need for such high potentials in half-cell experiments means that no electric work is available for the corresponding fuel cells and indicates the need to develop catalysts capable of oxidizing glycerol at lower potentials than those observed on Pt.

Taking into account the knowledge accumulated over the past decades about the mechanism of electrooxidation of alcohols similar to glycerol (such as ethanol), it is now widely accepted that a good electrocatalyst should have the skills of 
trapping the molecules on a surface and provide oxide species to promote the oxidation at potentials as low as possible $[7,8]$.

In this context, Lamy et al. showed that direct ethanol fuel cells (DEFC) perform best when Pt anodes are modified by tin [9]. The optimum composition was found to be in the range of $10-20 \%$ of tin [9]. The results were rationalized in terms of a lower CO poisoning, which is an intermediate of ethanol [9]. However, the authors also show that if those steps involving the dissociative adsorption of ethanol are hindered, products of intact chain as acetaldehyde and acetic acid are generated, undermining the production of $\mathrm{CO}_{2}[9]$.

Concerning the role played by Sn during the electrooxidation of ethanol on PtSn catalysts, there is no consensus among different authors. For instance, Colmati et al. suggest that PtSn catalysts favor the breaking of the $\mathrm{C}-\mathrm{C}$ bonds at lower potentials than those required for sole Pt [10]. On the other hand, Wang et al. conclude that the presence of Sn does not enhance the activity for $\mathrm{C}-\mathrm{C}$ bond breaking [11]. Their results are interpreted in terms of a higher yield of intact chain products instead of an improved formation of $\mathrm{CO}_{2}$ [11].

However, extrapolation of the results found for ethanol to more complex alcohols, such as glycerol, does not seem to be a wise strategy. For instance, in a recent work, we demonstrate that when similar amounts of ethanol and glycerol are electrooxidized on Pt nanoparticles, the production of $\mathrm{CO}_{2}$ (followed by in situ FTIR) is six times higher for glycerol than for ethanol, providing that the potential is higher than $1.1 \mathrm{~V}$ [6]. Moreover, in situ FTIR also suggests that unlike what is observed for ethanol, glycerol electrooxidation occurs via several pathways that contribute to the production of $\mathrm{CO}_{2}$, probably through the oxidation of carboxylic acids and other partially oxidized products, without forming CO. [6]. Such results suggest that the presence of oxyphilic species (such as $\mathrm{Sn}$ ) could create the conditions necessary to oxidize glycerol to $\mathrm{CO}_{2}$. Hence, aiming to improve the catalytic response of Pt-based surfaces toward glycerol electrooxidation, we prepared PtSn electrodeposits in three different atomic ratios and submit them to successive cycles in presence of glycerol. Eventual compositional changes were followed by X-ray photoelectron spectroscopy (XPS) analysis, while the electrocatalytic performance in presence of glycerol was accompanied by cyclic voltammetry. Our results suggest that by subjecting PtSn catalysts to voltammetric cycles, it is possible to change (and eventually to control) the electrocatalytic response and even the surface composition of these materials. Such findings are relevant since they open up the possibility of exploring a wide range of surface compositions in a single, easy-to-perform experiment, without the need of preparing one electrode at a time (as it is usually done).

\section{Experimental}

Solutions were prepared with Milli-Q water $(18.2 \mathrm{M} \Omega \mathrm{cm})$, glycerol $\left(1.0 \mathrm{~mol} \mathrm{~L}^{-1}\right)$, and $\mathrm{H}_{2} \mathrm{SO}_{4}\left(0.1 \mathrm{~mol} \mathrm{~L}^{-1}\right)$. Before the experiments, the solutions were thoroughly deaerated with $\mathrm{N}_{2}$. The counter electrode was a platinum sheet, and all the potentials were measured against an RHE in the same electrolyte. All the experiments were performed at room temperature $\left(25.0 \pm 1.0^{\circ} \mathrm{C}\right)$.

PtSn electrodes were prepared as electrodeposits on a polished gold disk. The metals were electrodeposited by electrochemical reduction of $\mathrm{Pt}^{4+}$ and $\mathrm{Sn}^{2+}$, obtained from $\mathrm{H}_{2} \mathrm{PtCl}_{6}$ and $\mathrm{SnCl}_{2} .2 \mathrm{H}_{2} \mathrm{O}$ (Sigma-Aldrich) aqueous solutions, at $0.08 \mathrm{~V}$ vs. RHE during $10 \mathrm{~min}$. Three distinct compositions were obtained by changing the relative amounts of $\mathrm{Pt}^{4+}$ and $\mathrm{Sn}^{2+}$. Real compositions of electrodeposits were estimated by EDX and XPS analyses.

For the estimation of the real surface area, the electrodeposits were saturated with carbon monoxide by bubbling the gas during $10 \mathrm{~min}$ at $0.05 \mathrm{~V}$. The excess of $\mathrm{CO}$ was then eliminated from the electrochemical cell by purging the solution with pure $\mathrm{N}_{2}$ during $30 \mathrm{~min}$. Afterward, three cyclic voltammograms were recorded at $0.02 \mathrm{~V} \mathrm{~s}^{-1}$ in the potential range of 0.05 $1.0 \mathrm{~V}$. The first cycle provided the total charge of $\mathrm{CO}$ oxidation, while the second and third ones were taken to check the recovery of the original voltammetric profile. It was assumed that the oxidation of one $\mathrm{CO}$ monolayer generates a charge of $420 \mu \mathrm{C} \mathrm{cm}^{-2}$ of real area of $\mathrm{Pt}$ ( $\mathrm{Sn}$ does not adsorb CO). For all experiments, electrodes were first kept at $0.15 \mathrm{~V}$ in $0.1 \mathrm{~mol} \mathrm{~L}^{-1}$ $\mathrm{H}_{2} \mathrm{SO}_{4}$ and glycerol was added into the cell to reach a concentration of $1.0 \mathrm{~mol} \mathrm{~L}^{-1}$. The reaction was conducted through 5 , 100 , and 500 voltammetric cycles at a scan rate of $0.05 \mathrm{~V} \mathrm{~s}^{-1}$.

\section{Results and Discussion}

\section{Determination of Atomic Composition of PtSn Electrodeposits}

Table 1 summarizes the results of EDX and XPS analyses performed on PtSn electrodeposits and make clear that the compositions show important discrepancies when the techniques are compared. Namely, for all catalysts, the platinum content is sensibly higher when measured by EDX than by XPS. Such differences can be attributed to the different features of both techniques; the escape depth of the photoelectron in XPS is less than in EDX [12], thus EDX provides the bulk atomic composition, whereas XPS shows those compositions closer to the surface. Therefore, this comparative analysis allows us to infer that the bulk of our samples is richer in platinum, while the surfaces of the electrodeposits are mainly composed by tin. 
Table 1 Catalysts composition determined by EDX and XPS

\begin{tabular}{ll}
\hline Bulk composition EDX (Pt:Sn at.\%) & Surface composition XPS (Pt:Sn at.\%) \\
\hline $87: 13$ & $28: 72$ \\
$75: 25$ & $24: 76$ \\
$65: 35$ & $19: 81$ \\
\hline
\end{tabular}

Knowing that the electrochemical response of a catalyst depends exclusively on its surface and that the voltammetric profiles reflect the surface composition rather than the bulk of the samples, we decided to identify our catalysts by atomic compositions determined by XPS. Therefore, hereafter, the binary catalysts will be denoted as PtSn 28:72, 24:76, and 19:81 (the nominal compositions were 90:10, 70:30, and 60:40, respectively).

\section{Glycerol Electrooxidation}

The electrocatalytic activity of PtSn electrodeposits toward glycerol electrooxidation was investigated by cyclic voltammetry at a scan rate of $0.05 \mathrm{~V} \mathrm{~s}^{-1}$ in solutions containing $1.0 \mathrm{~mol} \mathrm{~L}^{-1}$ glycerol $+0.1 \mathrm{~mol} \mathrm{~L}^{-1} \mathrm{H}_{2} \mathrm{SO}_{4}$. The results for the first five voltammetric cycles are shown in Fig. 1.

The current densities obtained on PtSn surfaces are sensibly smaller than those observed for Pt. Based on the results in Table 1, the poor catalytic response is probably related to a low content of Pt on the surface of PtSn, which considerably decreases the number of active sites available for adsorption of glycerol molecules, compromising the whole oxidation process.

The voltammetric behavior of polycrystalline $\mathrm{Pt}$ in $1.0 \mathrm{~mol} \mathrm{~L}^{-1}$ glycerol $+0.1 \mathrm{~mol} \mathrm{~L}^{-1} \mathrm{H}_{2} \mathrm{SO}_{4}$ presents features similar to those observed in previous papers for the stable response of $\mathrm{Pt}$ in diluted $[13,14]$ and concentrated glycerol solutions [15]. During the positive-going potential scan, a single oxidation peak is observed at $0.87 \mathrm{~V}$; afterward, the current diminishes. Just after the reversion of the potential scan $(1.0 \mathrm{~V})$, a reactivation of the surface is noticed and glycerol oxidation currents resume, peaking at $\sim 0.7 \mathrm{~V}$.

On the other hand, the voltammetric behavior observed for PtSn electrodeposits cannot be directly compared with results from literature since data available for glycerol on PtSn (supported on glassy carbon) refer to compositions richer in Pt than the ones investigated here [16]. Figure 1 shows no oxidation peaks for any of the binary catalysts, although an
Fig. 1 First voltammetric cycles performed on Pt and PtSn electrodes in $1.0 \mathrm{~mol} \mathrm{~L}^{-1}$ glycerol $+0.1 \mathrm{~mol} \mathrm{~L}^{-1} \mathrm{H}_{2} \mathrm{SO}_{4}$ at $0.05 \mathrm{~V} \mathrm{~s}^{-1}$. The compositions of the electrodeposits are indicated in the figure

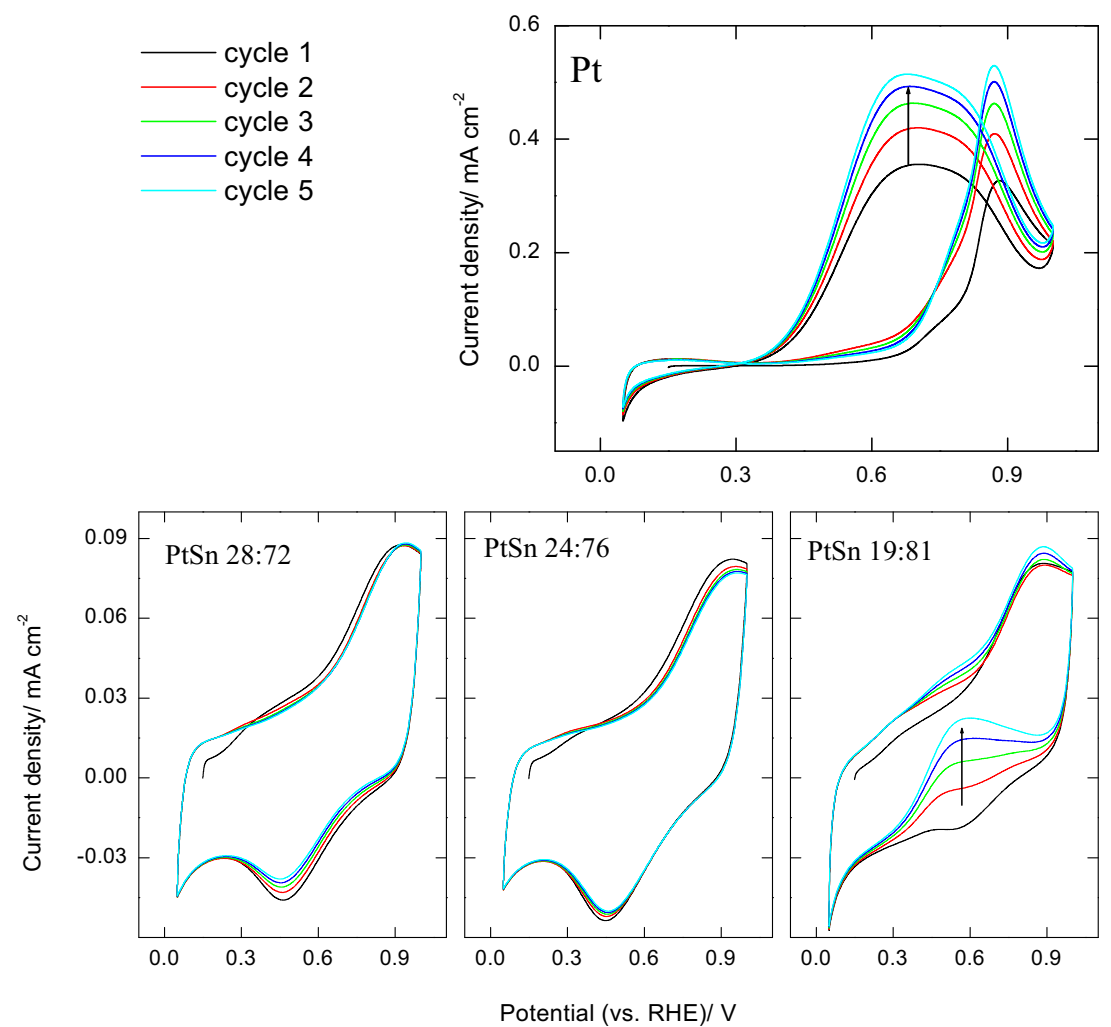


ill-defined shoulder can be perceived at high potentials during the positive-going scan, followed by the rising of cathodic currents in the reverse scan. Surprisingly, PtSn 19:81 is the catalyst which presents the first indication of anodic currents in the backward scan. Such behavior is intriguing, since this catalyst is the one who possesses less Pt on its surface, and its behavior is apparently the more similar to Pt. However, we must consider that the electrochemical active surface area (ECSA) of PtSn 19:81 is one order of magnitude lower than for the other catalysts. Considering that the areas of gold substrates were similar (they were taken from the same sample), such difference implies into different surface roughness. In this context, it is known that the surface roughness can bring deep implications into the mechanism of oxidation of a similar alcohol (ethanol) [17]. Namely, the rougher the surface, the easier is the production of species containing a single carbon atom (e.g., $\mathrm{CO}_{2}$ ) [17]. Results were rationalized in terms of a higher capability of rough electrodes to interact with adsorbed molecules, thus facilitating the scission of the carbon chain [17]. In the present study, Pt and PtSn 19:81 are the electrodes with smaller ECSA, while the areas of PtSn 28:72 and 24:76 are at least one order of magnitude higher (the specific areas will be presented later in the text). Such differences suggest that for those catalysts of smaller areas, the strength of interaction with the surface is not enough to guarantee a full oxidation cycle and some species are adsorbed and oxidized during the backward sweep. For those electrodes with big areas, the oxidation seems to happen at once. Another aspect that cannot be disregarded is the fact that although PtSn 19:81 is the binary surface richer in Sn, the initial loss of $\mathrm{Sn}$ could happen at higher rates, considering its small area. However, a complete description of the rate of composition change would involve the analysis of numerous catalysts, since these materials cannot be reused after XPS analysis.
Now, let us compare the initial voltammetric responses for the binary catalysts in the absence and presence of glycerol (Fig. 2). For all catalysts, the voltammetric blank responses (absence of glycerol, black lines) are similar, which merely reflect the fact that all surfaces have similar atomic compositions. In presence of glycerol, the catalysts show a minor oxidation shoulder at potentials close to $1.0 \mathrm{~V}$, while in the reverse scan, the reduction of tin oxides $(\sim 0.5 \mathrm{~V})$ is inhibited when compared to the corresponding blank voltammograms. Although similar, those features are more pronounced for PtSn 19:81, which are probably due to the fact that this catalyst has the higher rate of compositional change, as already discussed.

Overall, all binary catalysts show a very poor activity for glycerol electrooxidation (minor oxidation currents in presence of the alcohol), probably because there are not enough Pt sites on the surface to effectively promote glycerol adsorption-oxidation steps.

\section{Investigation of Stability and Activity of PtSn Electrodeposits Toward Glycerol Electrooxidation}

It is well known that voltammetric cycling in acidic media induces a progressive loss of $\mathrm{Sn}$ in $\mathrm{PtSn}$ catalysts, thus provoking a continuous change of surface composition in these materials [18]. Such effect has also been observed in shutdown tests performed on direct ethanol fuel cells [19]. With the aim to investigate further changes in the surfaces of Pt and PtSn, the electrodes were subjected to 100 and 500 voltammetric cycles, as shown in Figs. 3 and 4, respectively. The voltammetric studies were conducted with two independent series of fresh electrodes. The first ones were cycled 100 times, while the second group was cycled 500 times.
Fig. 2 Overlap of cyclic voltammograms obtained in presence (red line) and absence (black line) of glycerol $\left(1.0 \mathrm{~mol} \mathrm{~L}^{-1}\right)$ on PtSn surfaces. $v=0.05 \mathrm{~V} \mathrm{~s}^{-1}$ (color figure online)

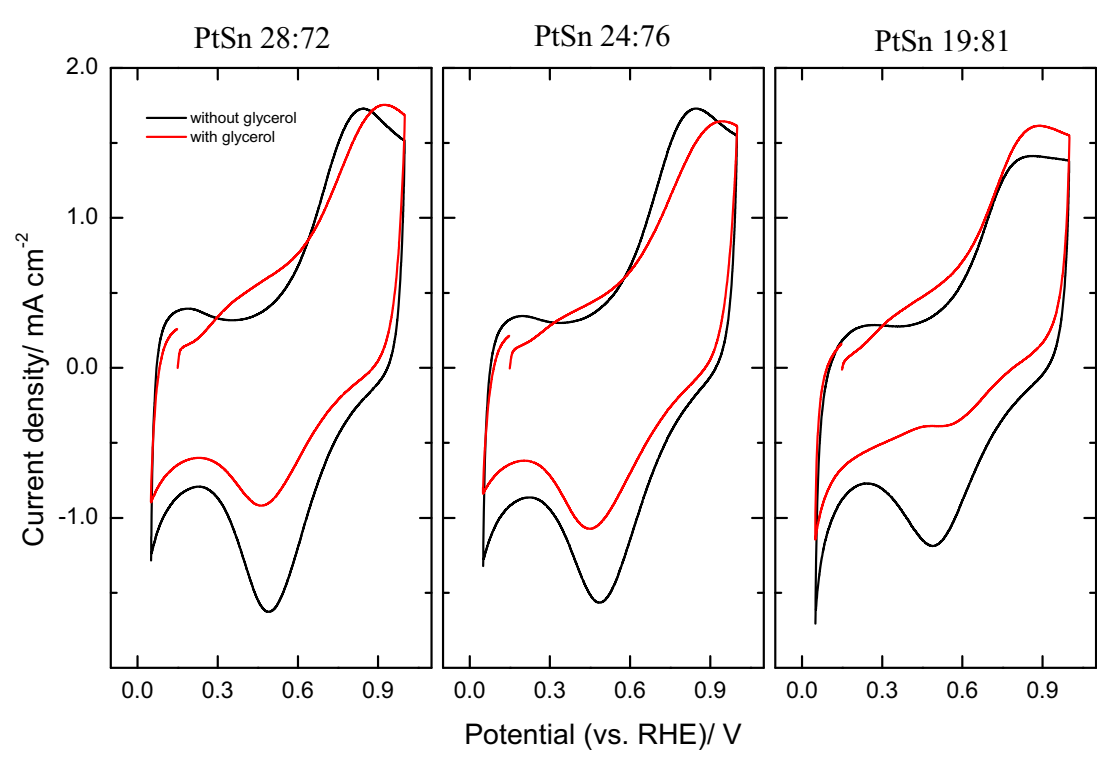


Fig. 3 Illustrative cyclic

voltammograms obtained during

100 cycles of glycerol

electrooxidation $\left(1.0 \mathrm{~mol} \mathrm{~L}^{-1}\right)$ on

$\mathrm{Pt}$ and PtSn catalysts.

$v=0.05 \mathrm{~V} \mathrm{~s}^{-1}$
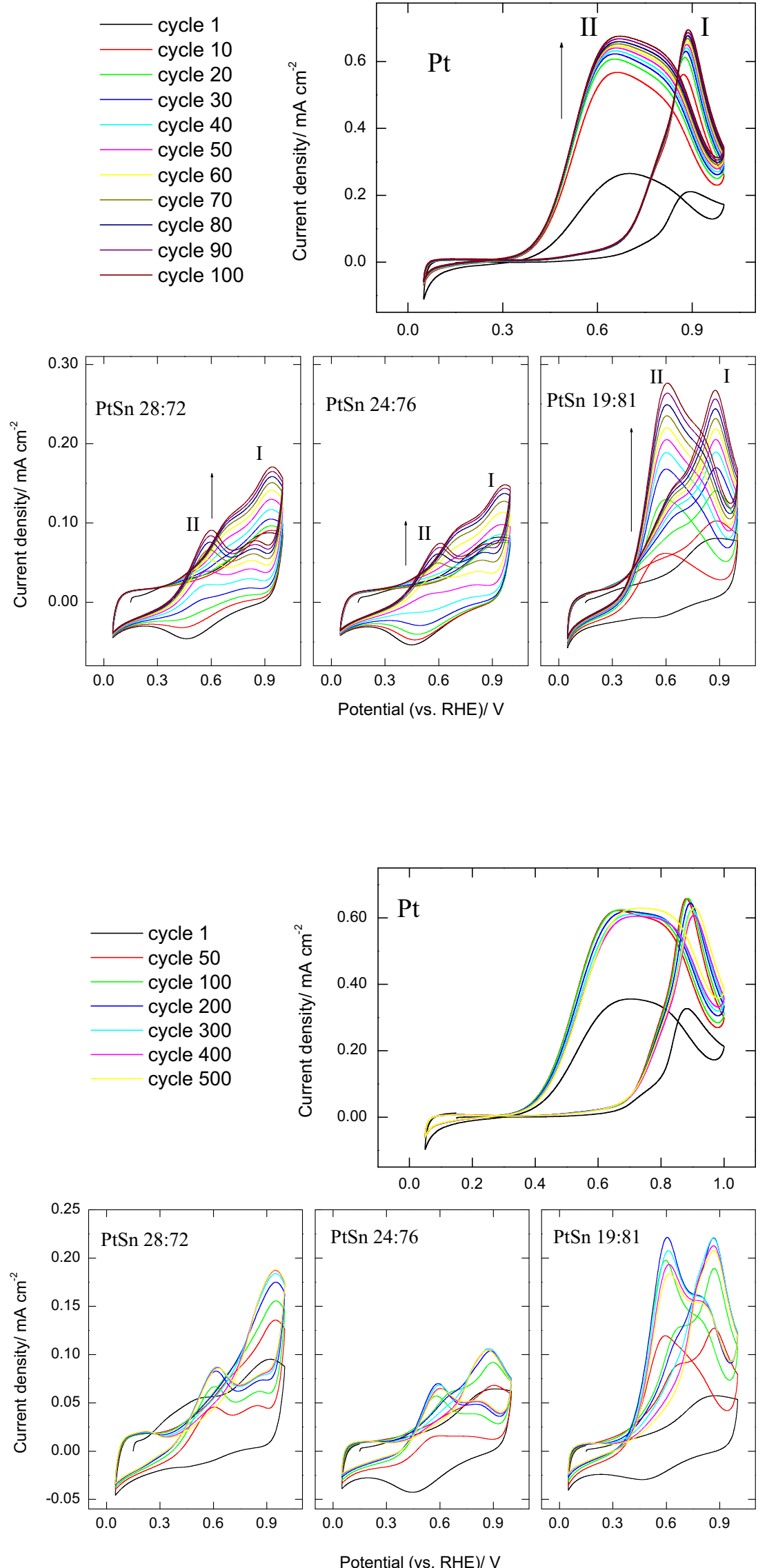

cycle 1

cycle 30

cycle 40

cycle 50

cycle 60

cycle 70

cycle 90

cycle 100

Potential (vs. RHE)/ V

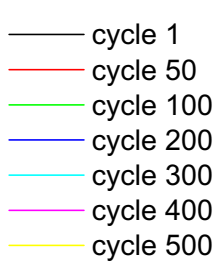

Potential (vs. RHE)/ V
Fig. 4 Illustrative cyclic voltammograms obtained during 500 cycles of glycerol electrooxidation $\left(1.0 \mathrm{~mol} \mathrm{~L}^{-1}\right)$ on $\mathrm{Pt}$ and PtSn catalysts. $v=0.05 \mathrm{~V} \mathrm{~s}^{-1}$ 
For all catalysts, we observe an oxidation peak during positive sweep and another peak in the negative sweep hereafter, denoted peaks I and II respectively. For PtSn electrodeposits, we observe that the current density continuously increases with the successive cycles until approximately the 200th and 300th cycle (Fig. 4) and then remains "stable" in subsequent cycles, while for $\mathrm{Pt}$, the current density is almost constant since the 50th cycle. In addition, from Figs. 3 and 4, we observe that as PtSn catalysts are cycled, their voltammetric responses increasingly resemble that of Pt. Namely, the shoulder preceding the oxidation peak (typical of PtSn) disappears while the single oxidation peak observed in positive scan (typical of $\mathrm{Pt}$ ) becomes more pronounced. If we presume that the shoulder is provoked by the presence of Sn on the electrodeposits surface, its disappearance reinforces that the surface composition is changing with the cycles, probably at the expenses of a loss of Sn.

Figures 3 and 4 also show how the electrochemical cycling strongly influences the electrooxidation of glycerol on all surfaces investigated. Beside the voltammetric features, the current densities are also progressively changing during cycling, especially for PtSn electrodes. Such results suggest that probably the ECSA is also changing as the system evolves in time. Hence, in order to have a more comprehensive understanding of what happens to $\mathrm{PtSn}$ surfaces as they are cycled, we used $\mathrm{CO}$ as a probe to characterize the surfaces before and after cycling, as shown and discussed in the next section.

\section{Surface Characterization After Successive Oxidation Cycles}

Since CO stripping voltammetry is usually applied to characterize the electrochemical surfaces of some materials used in catalysis, we performed $\mathrm{CO}$ stripping experiments before and after cycling the PtSn catalysts in order to understand how the cycling influences the ECSA of these materials. Furthermore, the voltammetric features in presence of $\mathrm{CO}$ allow detecting eventual changes in the surface composition since they manifest as changes in the voltammetric profiles of $\mathrm{CO}$ oxidation. In order to have not only qualitative information about the surface composition, we also performed XPS analysis in the fresh electrodes as well as in the "cycled" ones.

Figures 5 and 6 show CO stripping voltammetry of PtSn electrodeposits obtained before (upper panels) and after (lower panels) glycerol electrooxidation performed in 100 and 500

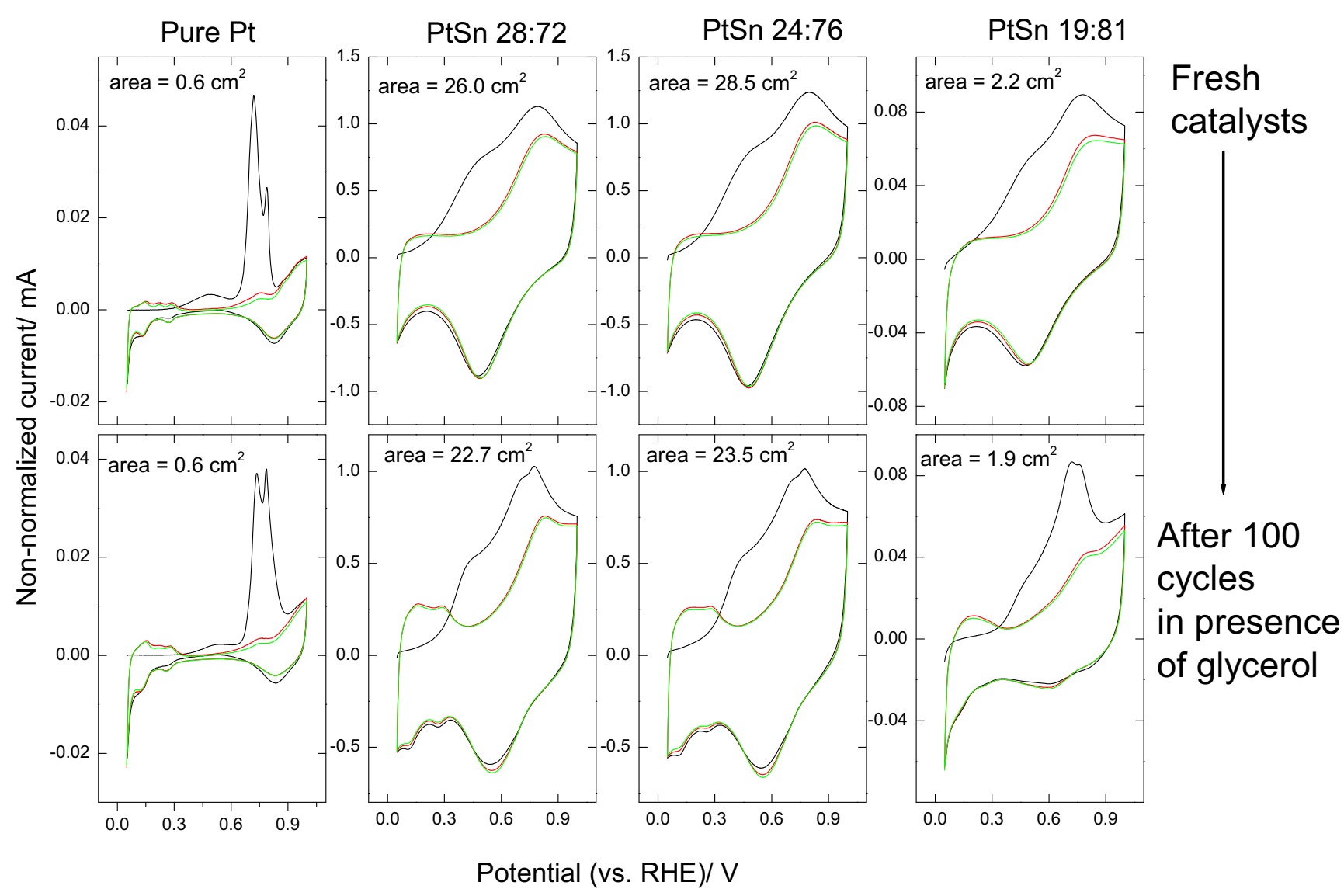

Fig. 5 CO stripping voltammetry performed on fresh Pt and PtSn catalysts (top panels) and after they were submitted to 100 voltammetric cycles in presence of $1.0 \mathrm{~mol} \mathrm{~L}^{-1}$ glycerol (bottom panels). $v=0.02 \mathrm{~V} \mathrm{~s}^{-1}$ 


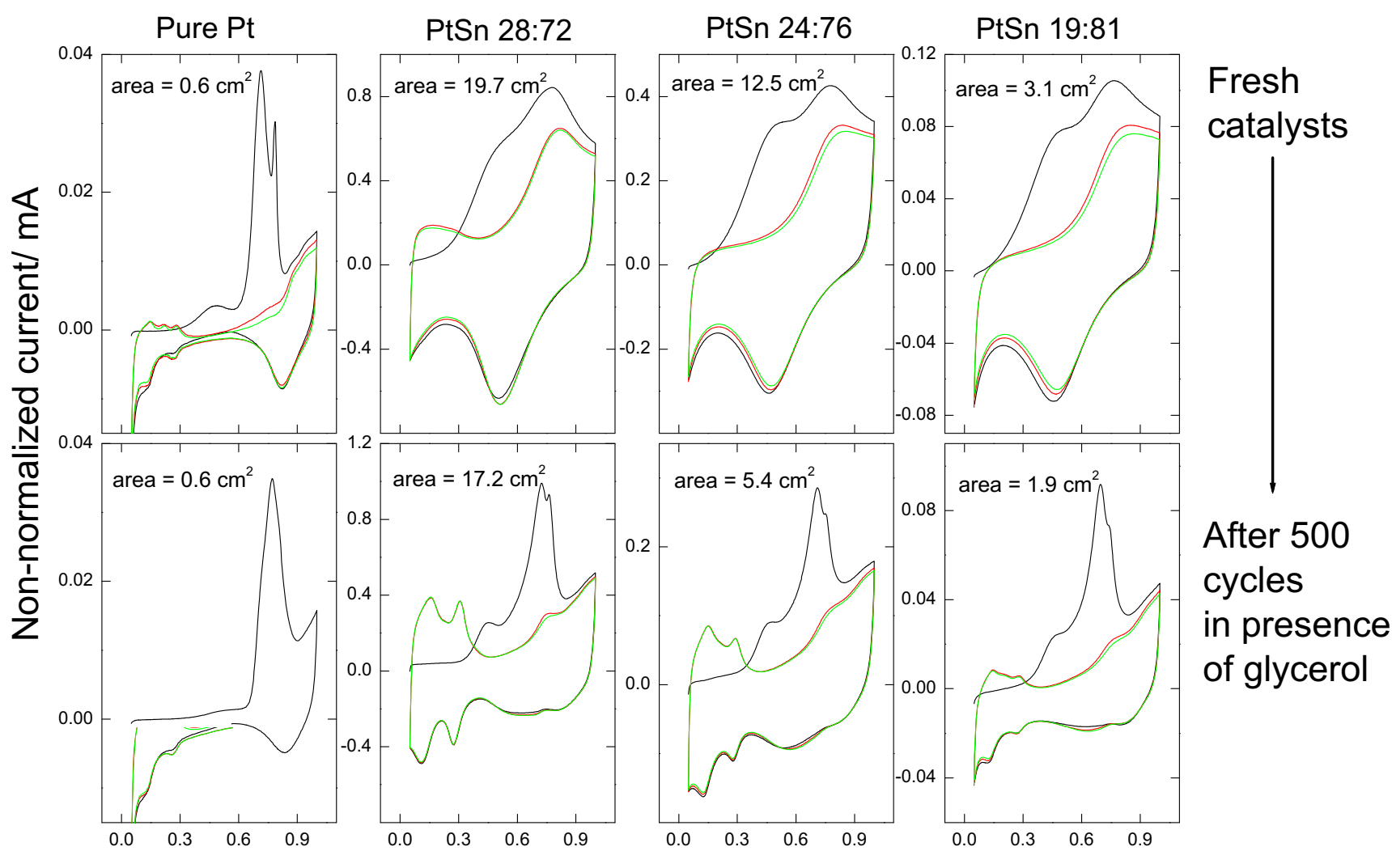

Potential (vs. RHE)/ V

Fig. 6 CO stripping voltammetry performed on fresh Pt and PtSn catalysts (top panels) and after they were submitted to 500 voltammetric cycles in presence of $1.0 \mathrm{~mol} \mathrm{~L}^{-1}$ glycerol (bottom panels). $v=0.02 \mathrm{~V} \mathrm{~s}^{-1}$

cycles, respectively. For purposes of comparison, results for pure $\mathrm{Pt}$ are also included. The corresponding areas are shown in graphs. In both figures, we note that $\mathrm{CO}$ is oxidized over a broad potential range with two poorly resolved peaks. Stevanovic et al. attributed the peak at low potentials to the Pt-Sn phase and the peak at higher potentials to the Pt phase [20].

As the electrodes are cycled 100 and 500 times (Figs. 5 and 6 , respectively), they become increasingly similar to Pt, both in terms of $\mathrm{CO}$ oxidation (black curve) and electrochemical profile after $\mathrm{CO}$ oxidation (red and green lines correspond to second and third cycles, respectively). The hydrogen adsorption/desorption peaks become well resolved and suggest that the Pt content on the surface increases as the electrodeposits are cycled. Moreover, we also observe that the $\mathrm{CO}$ oxidation peak becomes well defined as the electrodes are cycled, resembling the response observed for Pt.

These results confirm that important surface changes are taking place as the electrocatalysts are cycled and help us to understand the disappearance of the glycerol oxidation shoulder depicted in Fig. 4. Assuming that this "CO preoxidation" is due to the presence of Sn atoms [20], its disappearance indicates that surface Sn decreases with cycling. However, we cannot affirm that this phenomenon is solely provoked by Sn dissolution because the currents associated to the double layer region are still significantly large for PtSn, even after they are cycled (Figs. 5 and 6). This observation does not corroborate the assumption that the surface is "just losing Sn" (probably by dissolution), because if that was the case, those surfaces richer in Pt should present
Table 2 Electrochemical active surface area (ECSA) before and after glycerol electrooxidation $\left(1.0 \mathrm{~mol} \mathrm{~L}^{-1}\right)$ performed in 100 and 500 voltammetric cycles

\begin{tabular}{|c|c|c|c|c|c|c|}
\hline \multirow[t]{2}{*}{ PtSn electrodeposits } & \multicolumn{3}{|l|}{100 cycles } & \multicolumn{3}{|l|}{500 cycles } \\
\hline & Before $/ \mathrm{cm}^{2}$ & After $/ \mathrm{cm}^{2}$ & Reduction & Before $/ \mathrm{cm}^{2}$ & After $/ \mathrm{cm}^{2}$ & Reduction \\
\hline $28: 72$ & 26.03 & 22.68 & $12.9 \%$ & 19.66 & 17.18 & $12.9 \%$ \\
\hline $24: 76$ & 28.47 & 23.51 & $17.42 \%$ & 12.48 & 5.37 & $57 \%$ \\
\hline $19: 81$ & 2.2 & 1.91 & $13.2 \%$ & 3.12 & 1.86 & $40.4 \%$ \\
\hline
\end{tabular}


Fig. 7 Overview of glycerol electrooxidation $\left(1.0 \mathrm{~mol} \mathrm{~L}^{-1}\right)$ on PtSn surfaces. The figure shows cycles 1,100 , and 500 . $v=0.05 \mathrm{~V} \mathrm{~s}^{-1}$. Currents are normalized by the final ECSA

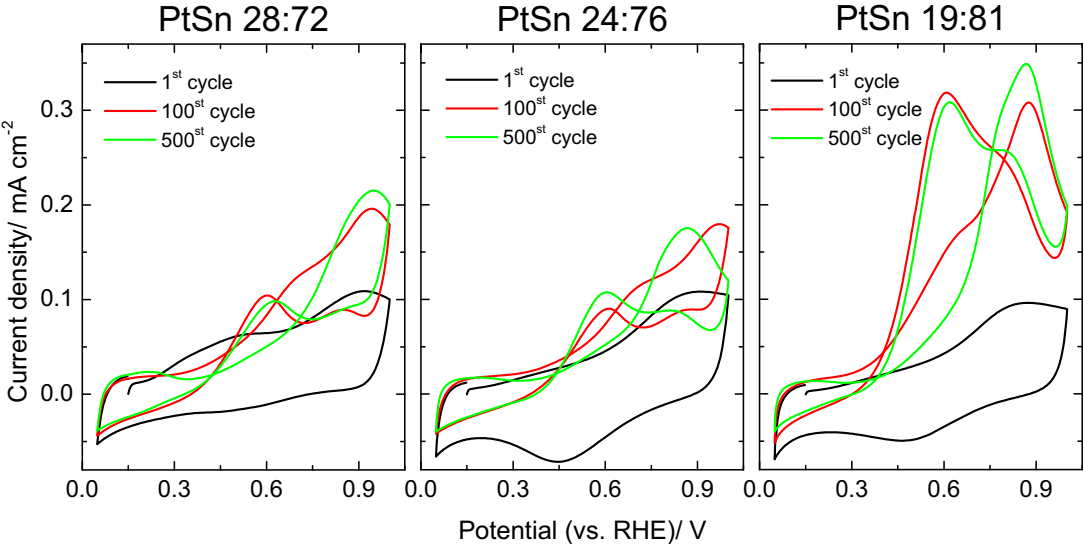

"narrower" double layer regions. Besides, the responses are clearly different from that of polycrystalline platinum.

Table 2 shows the ECSA estimated for the binary catalysts before and after the voltammetric cycles. It is important to note that fresh catalysts and those submitted to 100 and 500 cycles refer to different samples; hence, the surface areas and currents are not coincident. Overall, the cycling protocol results in a significant loss of ECSA for all catalysts, being this effect particularly evident for those surfaces richer in $\mathrm{Sn}$.

Here, it is reasonable to assume that PtSn electrodeposits may suffer a surface modification, but apparently, Sn keeps playing an active role in the electrooxidation of glycerol, despite its sensible displacement of the surface. The exact structure is beyond the scope of the present work.

Another important aspect of the current discussion is once we have established to begin with true fresh surfaces (including $\mathrm{Pt}$ ), the surfaces were not conditioned before the cycles depicted in Figs. 5 and 6. However, we are aware that some cleaning of the surface could explain the increase of currents observed during the first cycles, particularly for rough surfaces (Pt areas are virtually unaffected).

The results of Figs. 5 and 6 and Table 1 highlight the mistake that we commit in normalizing the currents of oxidation by the initial area of the catalysts (Figs. 3 and 4). For those figures, we cannot properly evaluate oxidation currents with successive cycling for the same electrodeposits since we do not have the areas in their respective cycles. Nonetheless, if we consider (i) that currents were normalized by the same factor (i.e., the initial electroactive area) and (ii) that ECSA decreases continuously with cycles for all catalysts, (iii) we can conclude that PtSn surfaces become intrinsically more active as they are cycled.

We illustrate this finding by using the following procedure: we normalize the current densities corresponding to the 100th and 500th cycles (Figs. 3 and 4, respectively) by the initial ECSA, in order to obtain a non-normalized current. Next, we divide these values by the final ECSA, as shown in Fig. 7. These results are even more encouraging than the previous ones and indicate that these materials tend to improve their activity as they are submitted to successive voltammetric cycles.

Here, it is important to point out that we are not assuming that $\mathrm{Sn}$ adsorbs $\mathrm{CO}$; hence, the surface area estimated by $\mathrm{CO}$ stripping refers only to $\mathrm{Pt}$. Regardless, our results can be understood on the basis of a mechanism similar to the formation of the "skeleton structure," as proposed by Stevanovic et al. [20]. According to these authors, a "skeleton" structure is formed after the dissolution of $\mathrm{Sn}$ from $\mathrm{PtSn} / \mathrm{C}$ (during $\mathrm{CO}$ annealing). Hence, the surface reconstructs and becomes very rough and full of defects. In this new surface, $\mathrm{Sn}$ is present only in the subsurface layers [20]. On the other hand, in the same work, the authors also showed that the degradation of these structures results in a significant loss of ECSA [20], such as we observe in the present case. Here, the loss of ECSA is particularly evident for those electrodes richer in Sn, as shown in Table 2.

Here, we do not have enough evidences to state that the formation of a "skeleton structure" is also occurring in our electrodeposits. It is more reasonable to assume that $\mathrm{PtSn}$
Table 3 Characterization of surface composition of PtSn electrodeposits before and after glycerol electrooxidation $\left(1.0 \mathrm{~mol} \mathrm{~L}^{-1}\right)$

\begin{tabular}{|c|c|c|c|c|c|c|c|c|c|}
\hline \multirow[t]{2}{*}{ PtSn } & \multicolumn{3}{|c|}{ As-prepared } & \multicolumn{3}{|c|}{ After 100 cycles } & \multicolumn{3}{|c|}{ After 500 cycles } \\
\hline & $\begin{array}{l}\% \mathrm{Pt} / \mathrm{Pt}+ \\
\mathrm{Sn}\end{array}$ & $\% \mathrm{Pt}^{\circ}$ & $\begin{array}{l}\% \mathrm{Sn}^{\%} / \\
\mathrm{Sn}_{\text {total }}\end{array}$ & $\begin{array}{l}\% \mathrm{Pt} / \mathrm{Pt}+ \\
\mathrm{Sn}\end{array}$ & $\% \mathrm{Pt}^{\circ}$ & $\begin{array}{l}\% \mathrm{Sn}^{\circ} / \\
\mathrm{Sn}_{\text {total }}\end{array}$ & $\begin{array}{l}\% \mathrm{Pt} / \mathrm{Pt}+ \\
\mathrm{Sn}\end{array}$ & $\% \mathrm{Pt}^{\circ}$ & $\begin{array}{l}\% \mathrm{Sn}^{\circ} / \\
\mathrm{Sn}_{\text {total }}\end{array}$ \\
\hline $28: 72$ & $28 \%$ & $52 \%$ & $2 \%$ & $49 \%$ & $72 \%$ & - & $91 \%$ & $71 \%$ & - \\
\hline $24: 76$ & $24 \%$ & $46 \%$ & $8 \%$ & $36 \%$ & $71 \%$ & - & $51 \%$ & $86 \%$ & - \\
\hline $19: 81$ & $19 \%$ & $39 \%$ & $11 \%$ & $30 \%$ & $71 \%$ & - & $67 \%$ & $81 \%$ & - \\
\hline
\end{tabular}

Data obtained from XPS analysis 
electrodeposits may suffer an analogous surface modification, in which Sn keeps playing an active role in the electrooxidation of glycerol, despite its sensible displacement of the surface. The exact structure is beyond the scope of the present work.

Beside the ECSA changes, cycling the electrodes implies another important surface modification: the compositions of the electrocatalysts and the oxidation states of the surface change with successive cycles. Table 3 shows the results of a XPS analysis performed after 100 and 500 cycles for all electrodeposits. Data were extracted by fitting the Pt4f and Sn3d signals.

For fresh electrodeposits between 40 and $50 \%$ surface, Pt is in the metallic state $\left(\mathrm{Pt}^{\circ}\right)$ while $\mathrm{Sn}$ is predominantly present in the oxidized form (over $90 \%$ is $\mathrm{SnO}_{\mathrm{x}}$ ). As the electrodeposits are cycled, they turn richer in $\mathrm{Pt}$, both in terms of $(\mathrm{Pt} / \mathrm{Pt}+\mathrm{Sn})$ ratio as in the percentage of $\mathrm{Pt}^{\circ}$. Consequently, metallic $\mathrm{Pt}$ becomes predominant on all surfaces after 100 cycles. It is noteworthy that although all the electrodeposits start from similar surface compositions, they evolve differently. Such differences are probably related to the fact that below the fresh surface, the composition is sensibly different for each electrode, as illustrated by the bulk compositions (EDX results) depicted in Table 1. Therefore, while each surface changes with the cycles, the magnitude of this change is mainly determined by the composition of those atoms which constitute the subsurface, resulting in different surface compositions. Regarding $\mathrm{Sn}$, the $\mathrm{Sn}^{\circ}$ amount decreases with the cycling protocol, becoming negligible after 100 cycles.

If we correlate the XPS results with Fig. 4, we observe that the catalytic activity increases as PtSn surfaces get richer in Pt. This result is explained as follows: fresh electrodes have few sites available to glycerol adsorption (the $\mathrm{Pt}^{\circ}$ content lies between 19 and $28 \%$ ), meaning that their surfaces are "blocked" by Sn, which is mostly present as Sn oxides, even in fresh surfaces. As the electrodes are cycled, they turn richer in $\mathrm{Pt}$ (mainly $\mathrm{Pt}^{\circ}$ ) so that there are more sites available for adsorption-oxidation steps. In other words, the catalytic activity of PtSn electrodeposits increases as they are cycled due to the increase of Pt content (at the expenses of Sn content). Thus, the surface composition eventually reaches an optimum compromise between those sites available for adsorption (Pt sites) and the ones which act as sources of oxygen to assist the oxidation (Sn atoms). Finally, Fig. 7 and Table 3 suggest that the best PtSn superficial composition for the electrooxidation of glycerol seems to lie around 65:35 (i.e., after 500 cycles being performed).

\section{Conclusions}

- Fresh PtSn electrodeposits prepared in acidic media present surface compositions sensibly richer in $\mathrm{Sn}$ than the corresponding bulk compositions.
- This initial "excess" of Sn probably limits the number of sites available to adsorb and electrooxidize glycerol. As a consequence, all materials investigated present a poor activity toward glycerol electrooxidation.

- As the PtSn electrodeposits are cycled, they suffer important and continuous surface modifications: (i) a reduction of the electrochemically active surface area and, more importantly, (ii) continuous changes in the surface composition and oxidation states of both metals. Namely, the surface becomes increasingly richer in $\mathrm{Pt}$ (mainly $\mathrm{Pt}^{\circ}$ ).

- As the surfaces become richer in Pt, more sites become available for adsorption-oxidation steps. The global output is that the catalytic activity of PtSn increases as they are cycled in presence of glycerol.

- Looking toward a broader perspective, our results suggest that catalytic surfaces with tunable features (such as surface composition and catalytic response) can be obtained by the application of easily executable electrochemical protocols.

Acknowledgments The authors thank CNPq (grant nos. 405695/20136 and 309176/2015-8), FUNDECT, CAPES, MINCyT, and FINEP for funding this study. G.A.B. Mello is indebted to CAPES for a doctorate fellowship. P.S. Fernández acknowledges CONICET for a post doctorate fellowship. M.E. Martins acknowledges Universidad Nacional de La Plata and CONICET (PIP 112-201101-00917).

\section{References}

1. C. A. G. Quispe, C. J. R. Coronado, J. A. Carvalho Jr., Glycerol: production, consumption, prices, characterization and new trends in combustion. Renew. Sust. Energ. Rev. 27, 475 (2013)

2. P. S. Fernández, M. E. Martins, G. A. Camara, New insights about the electro-oxidation of glycerol on platinum nanoparticles supported on multi-walled carbon nanotubes. Electrochim. Acta 66, 180 (2012)

3. J. F. Gomes, C. A. Martins, M. J. Giz, G. Tremiliosi-Filho, G. A. Camara, Insights into the adsorption and electro-oxidation of glycerol: self-inhibition and concentration effects. J. Catal. 301, 154 (2013)

4. L. Roquet, E. M. Belgsir, J.-M. Léger, C. Lamy, Kinetics and mechanisms of the electrocatalytic oxidation of glycerol as investigated by chromatographic analysis of the reaction products: potential and pH effects. Electrochim. Acta 39, 2387 (1994)

5. P. S. Fernández, C. A. Martins, M. E. Martins, G. A. Camara, Electrooxidation of glycerol on platinum nanoparticles: deciphering how the position of each carbon affects the oxidation pathways. Electrochim. Acta 112, 686 (2013)

6. C. A. Martins, P. S. Fernández, H. E. Troiani, M. E. Martins, G. A. Camara, Ethanol vs. glycerol: understanding the lack of correlation between the oxidation currents and the production of $\mathrm{CO}_{2}$ on $\mathrm{Pt}$ nanoparticles. J. Electroanal. Chem. 231, 717-718 (2014)

7. A. Kowal, M. Li, M. Shao, K. Sasaki, M. B. Vukmirovic, J. Zhang, N. S. Marinkovic, P. Liu, A. I. Frenkel, R. R. Adzic, Ternary Pt/Rh/ $\mathrm{SnO}_{2}$ electrocatalysts for oxidizing ethanol to $\mathrm{CO}_{2}$. Nat. Mater. 8, 325 (2009) 
8. M. Li, D. A. Cullen, K. Sasaki, N. S. Marinkovic, K. More, R. R. Adzic, Ternary electrocatalysts for oxidizing ethanol to carbon dioxide: making Ir capable of splitting $\mathrm{C}-\mathrm{C}$ bond. J. Am. Chem. Soc. 135, 132 (2013)

9. C. Lamy, S. Rousseau, E. M. Belgsir, C. Coutanceau, J.-M. Léger, Recent progress in the direct ethanol fuel cell: development of new platinum-tin electrocatalysts. Electrochim. Acta 49, 3901 (2004)

10. F. Colmati, E. Antolini, E. R. Gonzalez, Effect of temperature on the mechanism of ethanol oxidation on carbon supported Pt, PtRu and $\mathrm{Pt}_{3} \mathrm{Sn}$ electrocatalysts. J. Power Sources 157, 98 (2006)

11. H. Wang, Z. Jusys, R. J. Behm, Ethanol electro-oxidation on carbon-supported $\mathrm{Pt}$, PtRu and $\mathrm{Pt}_{3} \mathrm{Sn}$ catalysts: a quantitative DEMS study. J. Power Sources 154, 351 (2006)

12. S. Beyhan, C. Coutanceau, J.-M. Léger, T. W. Napporn, F. Kadrigan, Promising anode candidates for direct ethanol fuel cell: carbon-supported PtSn-based trimetallic catalysts prepared by Bonnemann method. Int. J. Hydrogen Energ. 38, 6830 (2013)

13. A. Falase, M. Main, K. Garcia, A. Serov, C. Lau, P. Atanassov, Electrooxidation of ethylene glycol and glycerol by platinumbased binary and ternary nano-structured catalysts. Electrochim. Acta 66, 295 (2012)

14. P. S. Fernández, M. E. Martins, C. A. Martins, G. A. Camara, The electro-oxidation of isotopically labeled glycerol on platinum: new information on $\mathrm{C}-\mathrm{C}$ bond cleavage and $\mathrm{CO}_{2}$ production. Electrochem. Commun. 15, 14 (2012)

15. H. J. Kim, S. M. Choi, S. Green, G. A. Tompsett, S. H. Lee, G. W. Huber, W. B. Kim, Highly active and stable PtRuSn/C catalyst for electrooxidation on ethylene glycol and glycerol. Appl. Catal. BEnviron. 101, 366 (2011)

16. L. Zheng, L. Xiong, Q. Liu, K. Han, W. Liu, Y. Li, K. Tao, L. Niu, S. Yang, J. Xia, Enhanced electrocatalytic activity for the oxidation of liquid fuels on PtSn nanoparticles. Electrochim. Acta 56, 9860 (2011)

17. M. J. Giz, G. A. Camara, G. Maia, The etanol electrooxidation reaction at rough PtRu electrodeposits: a FTIR study. Electrochem. Commun. 11, 1586 (2009)

18. E. Casado-Rivera, D. J. Volpe, L. Alden, C. Lind, C. Downie, T. Vázquez-Alvarez, A. C. D. Angelo, F. J. DiSalvo, H. D. Abruña, Electrocatalytic activity of ordered intermetallic phases for fuel cell applications. J. Am. Chem. Soc. 126, 4043 (2004)

19. S. C. Zignani, V. Baglio, J. J. Linares, E. R. Gonzalez, A. S. Aricò, Endurance study of a solid polymer electrolyte direct ethanol fuel cell based on a Pt-Sn anode catalyst. Int. J. Hydrogen Energ. 38, 11576 (2013)

20. S. Stevanovic, D. Tripkovic, V. Tripkovic, D. Minic, A. Gavrilovic, A. Tripkovic, V. M. Jovanovic, Insight into the effect of Sn on CO and formic acid oxidation at PtSn catalysts. J. Phys. Chem. C 118, 278 (2014) 